\title{
Erratum to: Impact of a Blended Web-Based Mindfulness Programme for General Practitioners: a Pilot Study
}

\author{
Jesus Montero-Marin ${ }^{1,2}$ • Jorge Gaete ${ }^{3,4} \cdot$ Ricardo Araya $^{5}$ - Marcelo Demarzo ${ }^{6,7}$. $^{2}$ \\ Rick Manzanera $^{8}$ - Melchor Álvarez de Mon ${ }^{9}$ - Javier García-Campayo ${ }^{2,10}$
}

Published online: 3 August 2017

(C) Springer Science+Business Media, LLC 2017

Erratum to: Mindfulness (2017)

https://doi.org/10.1007/s12671-017-0752-8

The affiliation of the $2^{\text {nd }}$ author, Jorge Gaete, was misstated.

The correct affiliation is given below.

The online version of the original article can be found at https://doi.org/ $10.1007 / \mathrm{s} 12671-017-0752-8$

Jesus Montero-Marin

jmontero@unizar.es

1 Faculty of Health and Sport Sciences, University of Zaragoza, Huesca, Spain

2 Primary Care Prevention and Health Promotion Research Network (RedIAPP), Zaragoza, Spain

3 Department of Population Health, London School of Hygiene and Tropical Medicine, London, UK

4 Department of Public Health and Epidemiology, Faculty of Medicine, Universidad de los Andes, Santiago, Chile
5 Institute of Psychiatry, King's College, London, UK

6 Mente Aberta - Brazilian Center for Mindfulness and Health Promotion, Department of Preventive Medicine, Universidade Federal de São Paulo (UNIFESP), São Paulo, Brazil

7 Hospital Israelita Albert Einstein, São Paulo, Brazil

8 Primary Care \& Mental Health NHS, London, UK

9 University of Alcalá de Henares (UAH), Madrid, Spain

10 Miguel Servet University Hospital, University of Zaragoza, Zaragoza, Spain 